Accuracy tests of the tessellated SLBM model

Abelardo L. Ramirez, Stephen C. Myers

July 23, 2007 
This document was prepared as an account of work sponsored by an agency of the United States Government. Neither the United States Government nor the University of California nor any of their employees, makes any warranty, express or implied, or assumes any legal liability or responsibility for the accuracy, completeness, or usefulness of any information, apparatus, product, or process disclosed, or represents that its use would not infringe privately owned rights. Reference herein to any specific commercial product, process, or service by trade name, trademark, manufacturer, or otherwise, does not necessarily constitute or imply its endorsement, recommendation, or favoring by the United States Government or the University of California. The views and opinions of authors expressed herein do not necessarily state or reflect those of the United States Government or the University of California, and shall not be used for advertising or product endorsement purposes.

This work was performed under the auspices of the U.S. Department of Energy by University of California, Lawrence Livermore National Laboratory under Contract W-7405-Eng-48. 


\title{
ACCURACY TESTS OF THE TESSELLATED SLBM MODEL
}

\author{
Abelardo Ramirez and Stephen Myers \\ Lawrence Livermore National Laboratory \\ Livermore, California \\ July 19,2007
}

\section{Introduction}

We have compared the Seismic Location Base Model (SLBM) tessellated model (version 2.0 Beta, posted July 3, 2007) with the GNEMRE Unified Model. The comparison is done on a layer/depth-by-layer/depth and layer/velocity-by-layer/velocity comparison.

The SLBM earth model is defined on a tessellation that spans the globe at a constant resolution of about 1 degree (Ballard, 2007). For the tests, we used the earth model in file "unified_iasp.grid". This model contains the top 8 layers of of the Unified Model (UM) embedded in a global IASP91 grid. Our test queried the same set of nodes included in the UM model file.

To query the model stored in memory, we used some of the functionality built into the SLBMInterface object. We used the method getInterpolatedPoint() to return desired values for each layer at user-specified points. The values returned include: depth to the top of each layer, layer velocity, layer thickness and (for the upper-mantle layer) velocity gradient. The SLBM earth model has an extra middle crust layer whose values are used when $\mathrm{Pg} / \mathrm{Lg}$ phases are being calculated. This extra layer was not accessed by our tests.

\section{Test Results}

Figures 1 to 8 compare the layer depths, $\mathrm{P}$ velocities and $\mathrm{P}$ gradients in the $\mathrm{UM}$ and SLBM models. The figures show results for the three sediment layers, three crustal layers and the upper mantle layer defined in the UM model. Each layer in the models (sediment1, sediment2, sediment3, upper crust, middle crust, lower crust and upper mantle) is shown on a separate figure. The upper mantle $\mathrm{P}$ velocity and gradient distribution are shown on Figures 7 and 8.

The left and center images in the top row of each figure is the rendering of depth to the top of the specified layer for the UM and SLBM models. When a layer has zero thickness, its depth is the same as that of the layer above. The right image in the top row is the difference between in layer depth for the UM and SLBM renderings. The left and center images in the bottom row of the figures are renderings of the P velocity distribution within the specified layer for UM and SLBM models. The bottom right frame shows the difference in P velocity between UM and SLBM renderings. Black denotes zero thickness in both the UM and SLBM models. When the layer thickness is zero for one model and non-zero for the other model, then the velocity difference equals the velocity in the model with non-zero layer thickness. 


\section{Conclusions}

The figures suggest that most differences between the UM and SLBM models are small. The depth and velocity patterns in the figures are very similar. We can see some model differences at the edges of tectonic features. These are likely caused by the bilinear interpolation required to produce the tessellated model. Differences are accentuated North of 75 degrees latitude. These are probably due to the different resolution of the UM and SLBM models near the poles; the UM model is defined at a higher resolution close to the poles whereas the SLBM model is defined at an approximately constant resolution throughout the globe. In summary, our tests suggest that the SLBM model stored in memory for travel time calculation is sufficiently accurate for project needs.

\section{ACKNOWLEDGEMENTS}

We would like to thank Sandy Ballard for his guidance on how to access the functionality offered by the SLBMInterface object. We would also like to thank M. Pasyanos for his suggestions on how to compare the two models

\section{REFERENCES}

Ballard, Sanford, (2007). Seismic Location Base Model Software Design Document Version 2.0 Alpha, Sandia National Laboratories, July 3, 2007.

\section{FIGURES}




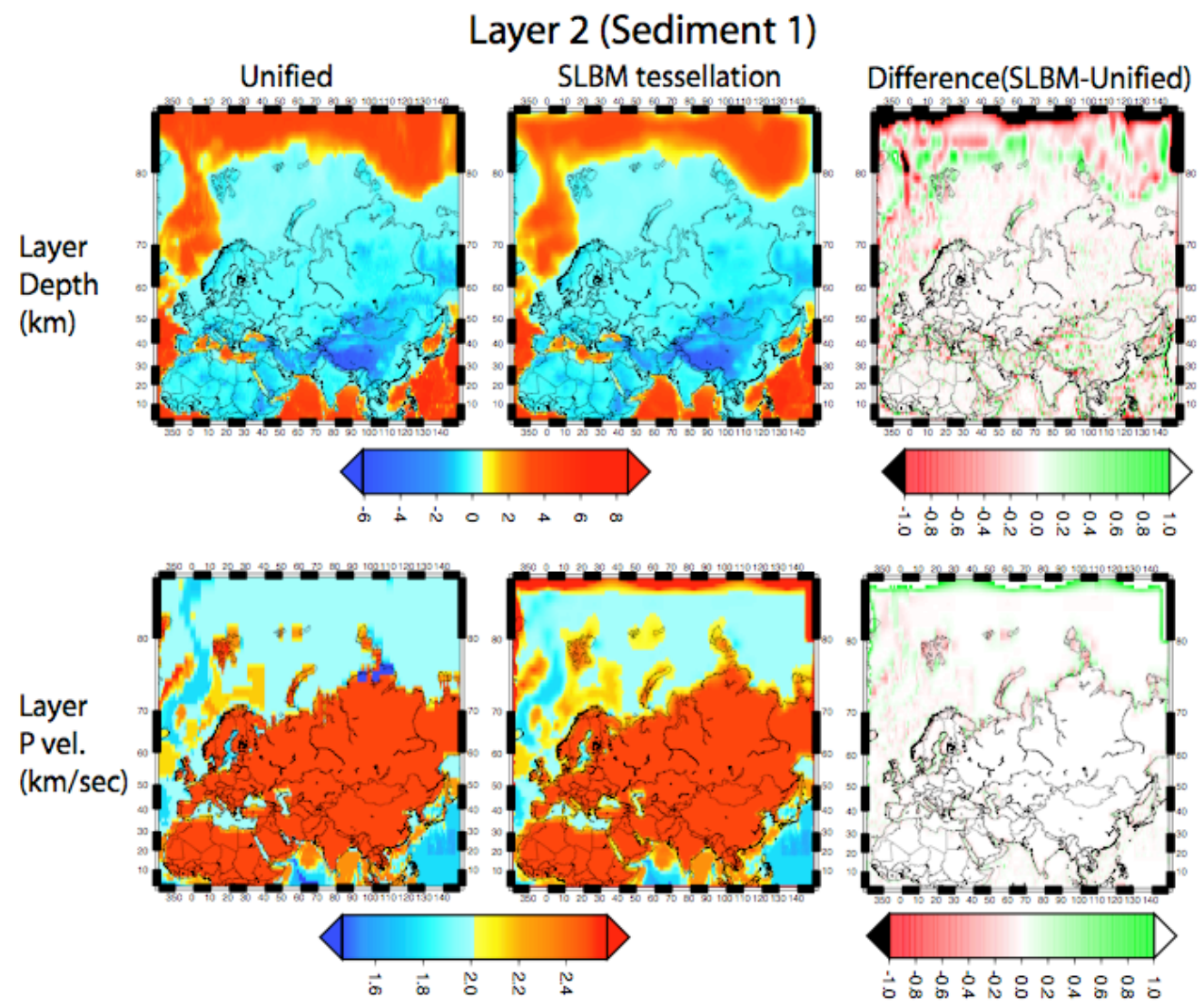

Figure 1 compares depths to the top of the sediment 1 layer (top row of images) and velocities (bottom row). The two leftmost image columns show absolute values and the right most images show differences. 


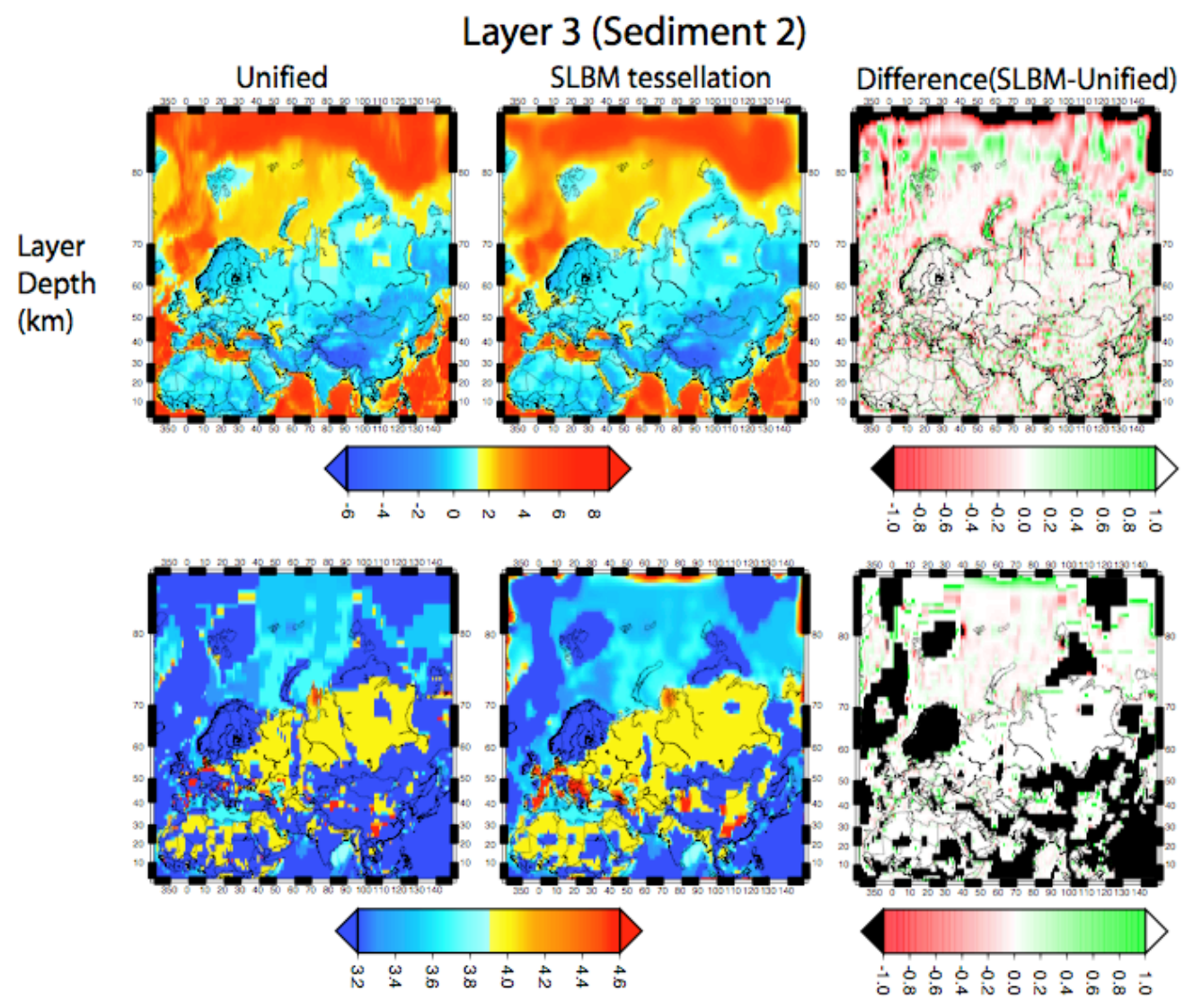

Figure 2 compares depths to the top of the sediment 2 layer (top row of images) and velocities (bottom row). Black regions in the velocity difference image (right, bottom image) indicate that the layer has zero thickness in both models. 


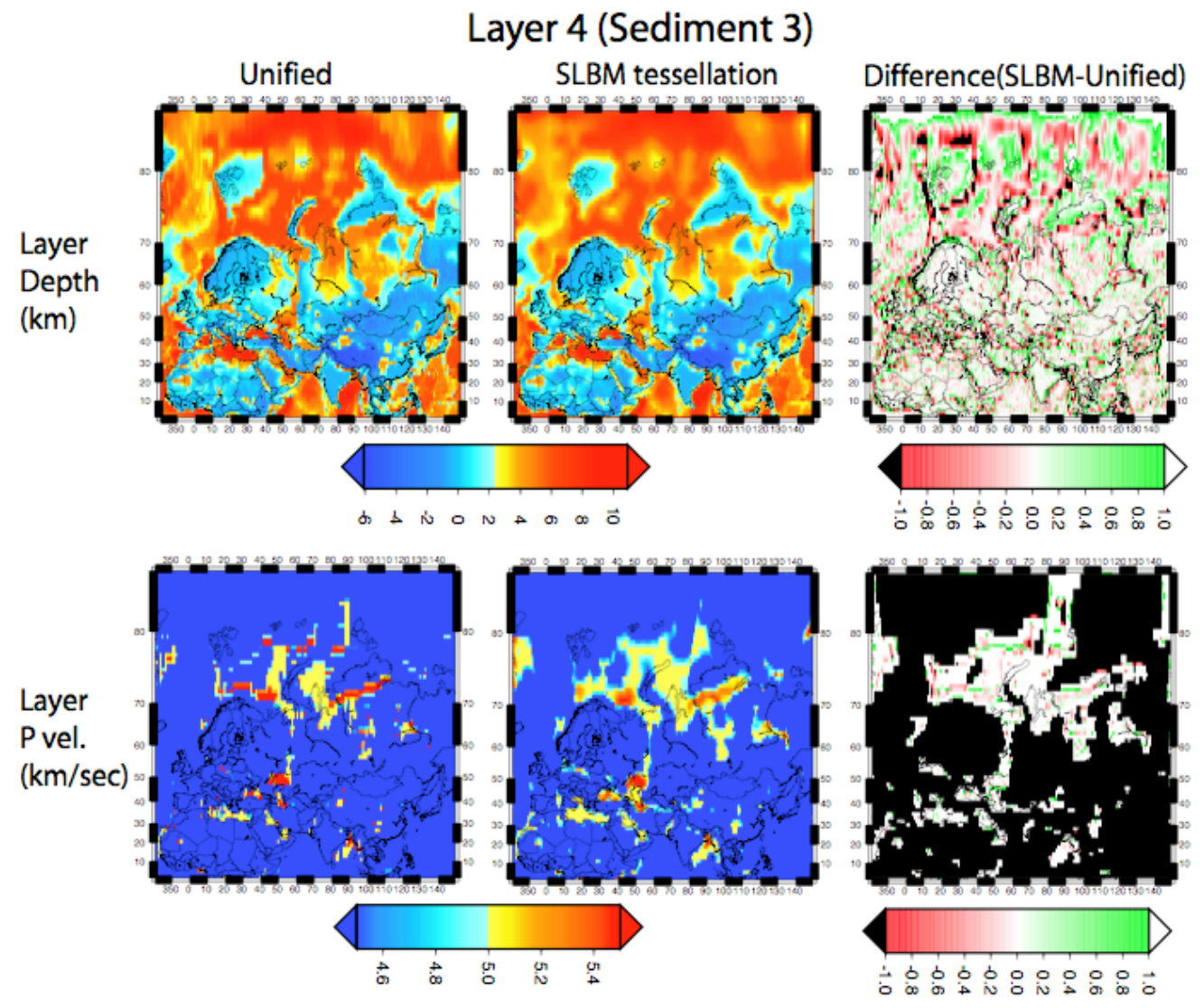

Figure 3 compares depths to the top of the sediment 3 layer (top row of images) and velocities (bottom row). Black regions in the velocity difference image (right, bottom image) indicate that the layer has zero thickness in both models. 


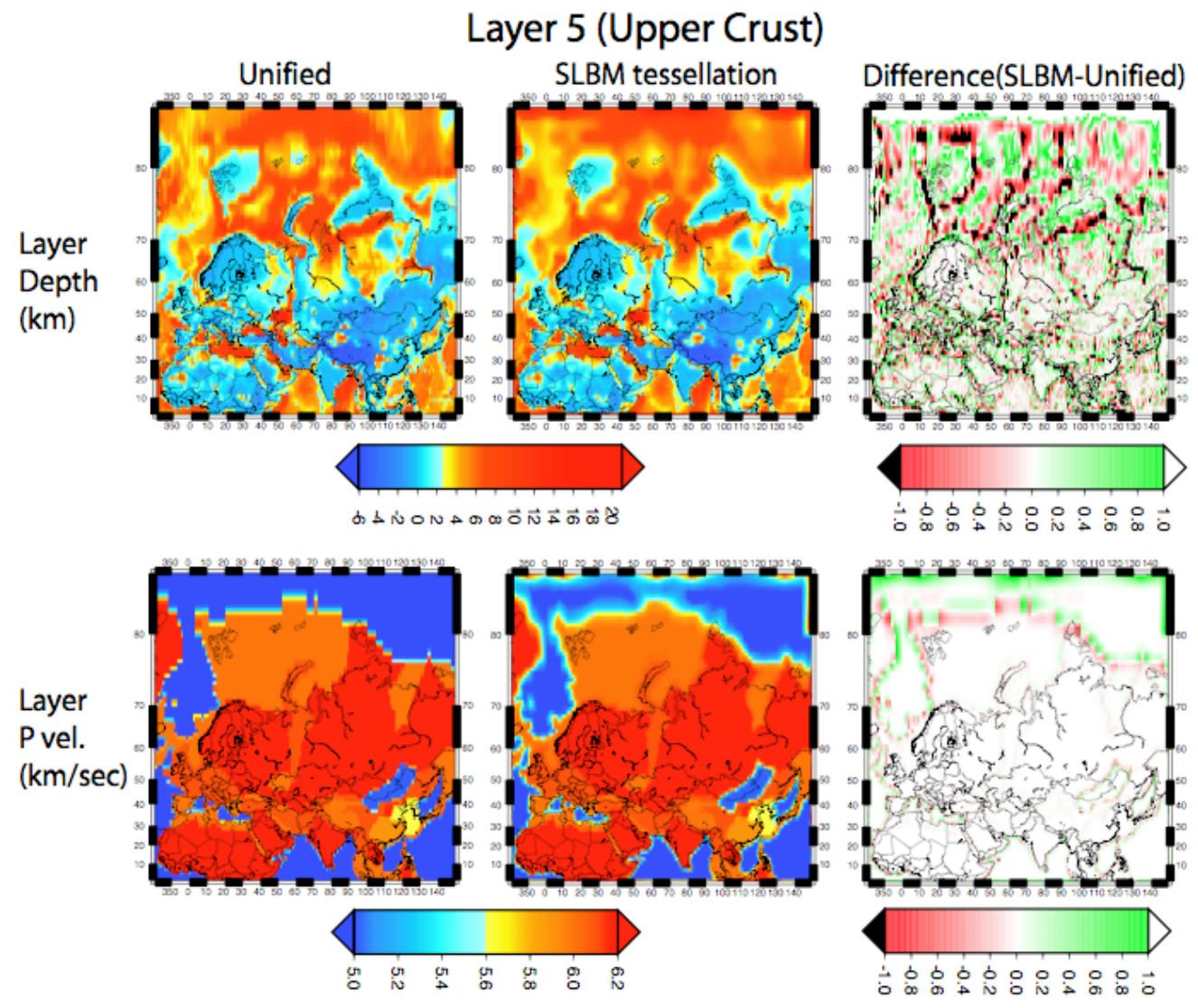

Figure 4 compares depths to the top of the upper crust layer (top row of images) and velocities (bottom row). The two leftmost image columns show absolute values and the right most images show differences. 


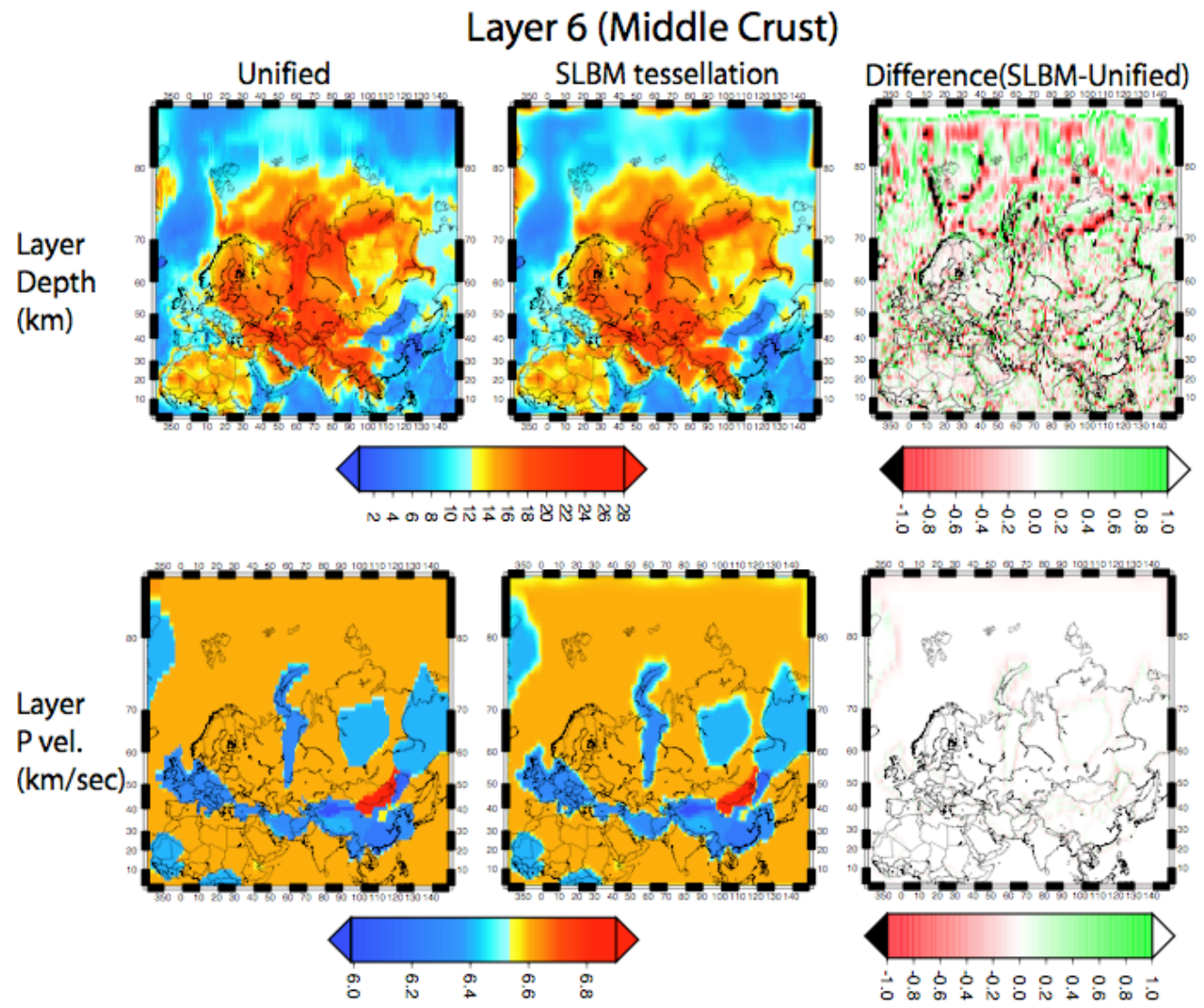

Figure 5 compares depths to the top of the middle crust layer (top row of images) and $\mathrm{P}$ velocities (bottom row). The two leftmost image columns show absolute values and the right most images show differences. 


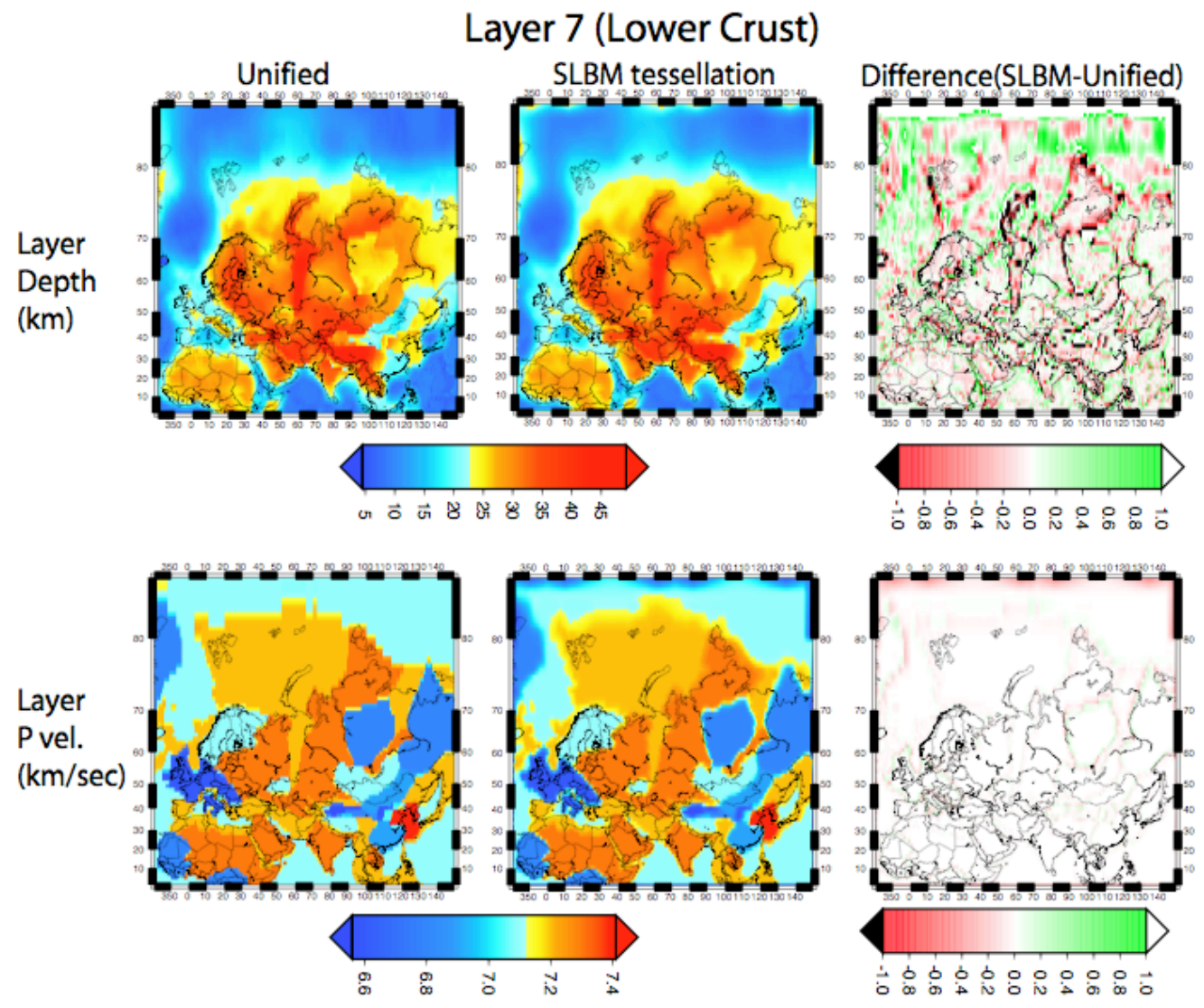

Figure 6 compares depths to the top of the lower crust layer (top row of images) and velocities (bottom row). The two leftmost image columns show absolute values and the right most images show differences. 


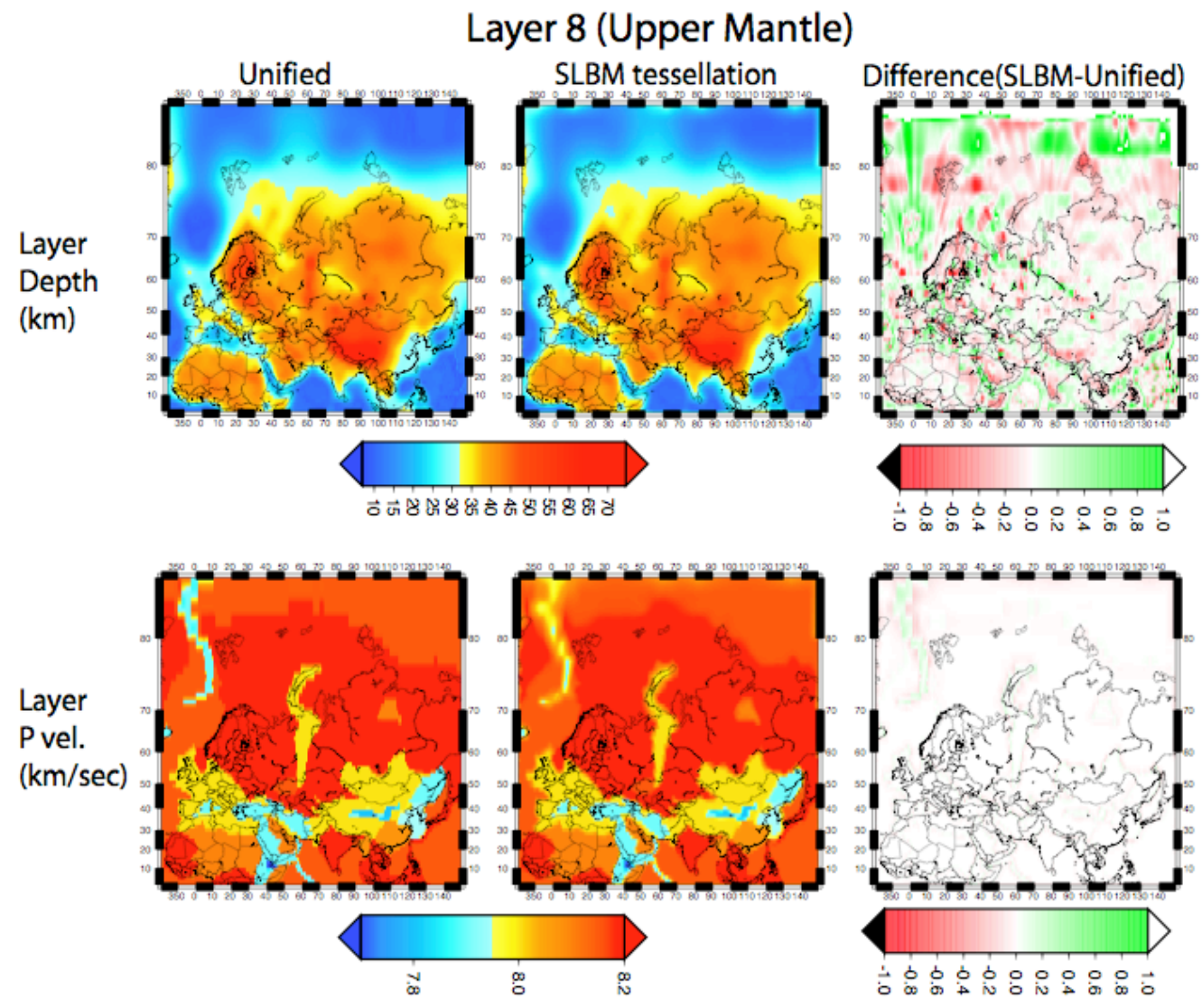

Figure 7 compares depths to the top of the upper mantle layer (top row of images) and velocities (bottom row). The two leftmost image columns show absolute values and the right most images show differences.

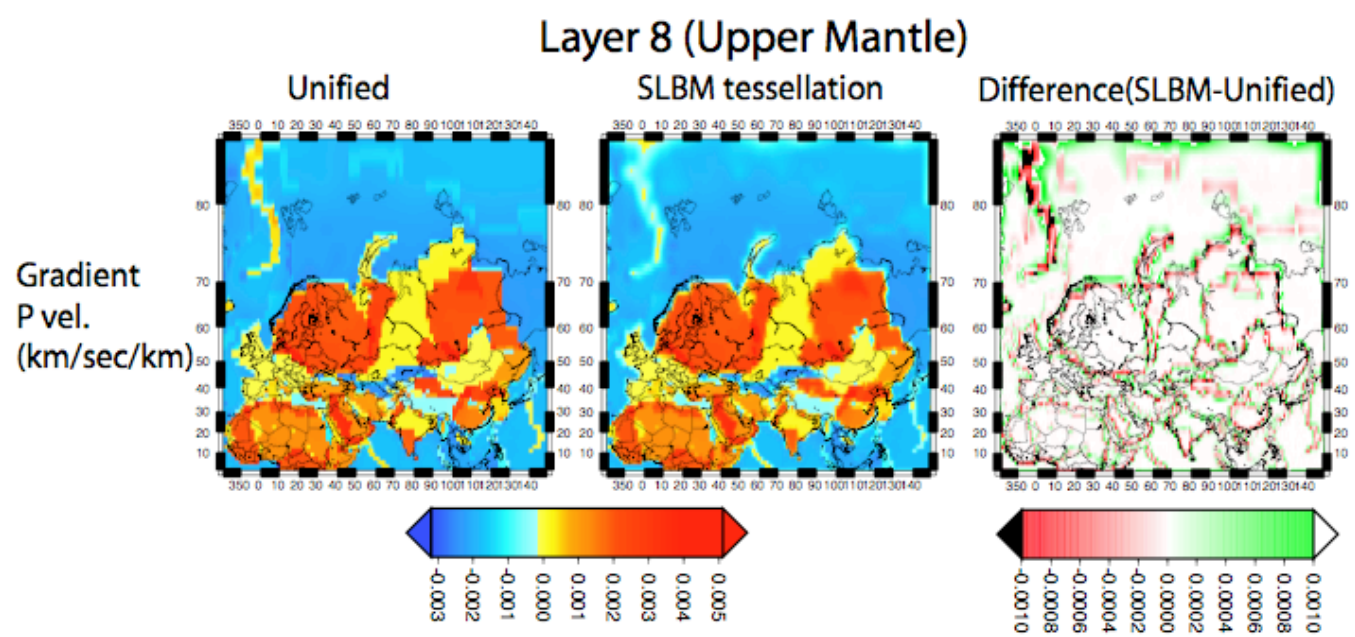

Figure 8 compares the $\mathrm{P}$ velocity gradients of the upper mantle layer. The right image shows gradient differences. 